\title{
The effect of macro and micro-nutrient fortification of cookies on their sensory properties and on hedonic liking of older people
}

\author{
R. Tsikritzi ${ }^{1}$, Y. Mavrommatis ${ }^{1}$, P. J. Moynihan ${ }^{2}$, M. A. Gosney ${ }^{3}$ and L. Methven ${ }^{1}$ \\ ${ }^{1}$ Department of Food and Nutritional Sciences, University of Reading, Reading RG6 6AP, UK, ${ }^{2}$ Institute for Ageing and \\ Health, Newcastle University, Framlington Place, Newcastle upon Tyne, NE2 4BW, UK and ${ }^{3}$ Clinical Health Sciences, \\ University of Reading, London Road, Reading, Berkshire, RG1 5AQ,UK
}

\begin{abstract}
Older adults can often have low food intake which increases risk of malnutrition and subsequent health issues. Oral nutritional supplement beverages are often subscribed, however older adults can find it difficult to consume oral nutritional supplement, to the extent that, even with feeding-support by trained staff, their nutritional status is not improved ${ }^{(1)}$. A variety of supplements with different sensory characteristics (appearance, flavour, texture, consistency and composition) is likely to improve compliance and intake to a greater extent than where only one type of supplement is used, especially for longer-term use when 'taste fatigue' can develop ${ }^{(2)}$. Meal fortification can improve energy $(+26 \%)$ and protein $(+23 \%)$ intake in hospitalised older adults ${ }^{(3)}$. This study successfully produced macronutrient dense oat cookies, and gluten free cookies, with a minimum energy content of $1874.43 \mathrm{~kJ} / 100 \mathrm{~g}$ and over $11 \%$ protein, comparing to typical short dough cookies of equivalent calorific density but lower protein content (7\%). Micronutrients were incorporated into the oat cookies by two approaches. The first was to add a full range of vitamins and minerals to replicate the approach taken by typical oral nutritional supplement beverages, where one portion usually provides from 9 to $69 \%$ of the recommended daily nutrient intake RNI of each nutrient. The second approach was to add micronutrients that were customised to the needs of older adults in hospital (OP) ${ }^{(4,5)}$; these cookies were enriched with Fe, riboflavin, vitamin B6, folic acid, vitamin C and vitamin D only, at a level of half the recommended daily nutrient intake per portion. Using a trained sensory panel to evaluate the cookies, no differences in basic tastes were found between the micronutrient enriched cookies from either approach in comparison to the control oat cookie, there were small but significant $(P<0.05)$ differences in some sensory attributes; however, these appeared to be due to batch to batch baking variation rather than due to the mineral fortification. Gluten free option was also prepared with $1874.43 \mathrm{~kJ} / 100 \mathrm{~g}$ and over $11 \%$ protein without micronutrient supplementation. In addition, when thirty-six older volunteers participated in hedonic liking there was no statistical difference in the mean liking of any of the cookies $(P=0.55)$. In conclusion, protein and micronutrient fortification of cookies appears to be a viable route to provide an alternative to oral nutritional supplement beverages, and a gluten free option would be possible. Data to date suggest that the micronutrient fortification could either be of all nutrients required daily, or a fortification tailored to the needs of older adults which considers the nutrients the group are most likely to be deficient in and/or have a low intake of; both routes led to acceptable products.
\end{abstract}

Study was sponsored by the New Dynamics of Ageing programme through ESRC, as part project 'mappmal'; developing new approaches to improving the nutrition of older people in hospitals. LycoRed are thanked for the development and supply of micronutrient premixes.

1. Milne AC et al. (2005) Nestle Nutrition Workshop Ser Clin Perform Programme. 10, 103-120.

2. Nieuwenhuizen WF et al. (2010) Clin Nutr 29, 160-169.

3. Gall MJ et al. (1998) Clin Nutr 17, 259-264.

4. Bates CJA et al. (1999) Br J Nutr 82, 7-15.

5. Russell RM \& Suter PM (1993) Am J Clin Nutr 58, 4-14. 